\title{
Examining Routing Methods and the Role of Neural Network in Wireless Sensor Networks.
}

\author{
Javad Nikafshar \\ M.A degree, information technology and computer networks
}

\begin{abstract}
A wireless sensor network is a set of numerous sensor networks with small dimensions and limited computing and tele-communicative capabilities used for collecting and transferring data from one environment toward a user of base station. Artificial neural network is a large system of parallel or distributed components called neuron or nerve cell connected to each other in a graph topology. In other words, artificial neural networks are math algorithms able to learn complex mappings between input(s) and output(s) through supervised training or capable of classifying input data by an unsupervised approach. There are several types of neural networks which each of them have various capabilities according to their usage.
\end{abstract}

Keywords: Wireless sensor network, neural network, neuron, topology

\section{Introduction}

One of the main controversial issues in wireless sensor networks is how data is transferred from nodes in the network to base station as well as choosing the best feasible route for transmitting these data. Selecting the best route can be affected by various factors including energy consumption, response speed and delay, and accuracy in data transfer. As the name of the article indicates, our purpose is to choose the best route in terms of energy consumption. Therefore, first, the concept of routing in these networks would be defined and then, some of the routing methods proposed for them will be concisely introduced.

\section{The Concept of Routing in Wireless Sensor Networks}

The way data and inquiries are transferred between the base station and the place the target phenomenon is seen is a highly important issue for wireless sensor networks called routing. In one viewpoint, routing can be considered as an approach of data transfer among sensor nodes. In another viewpoint, it can be defined as data transfer between the sensor nodes in the network and the final base station. Represented in figure 1 is the way the data are propagated in the wireless sensor network.

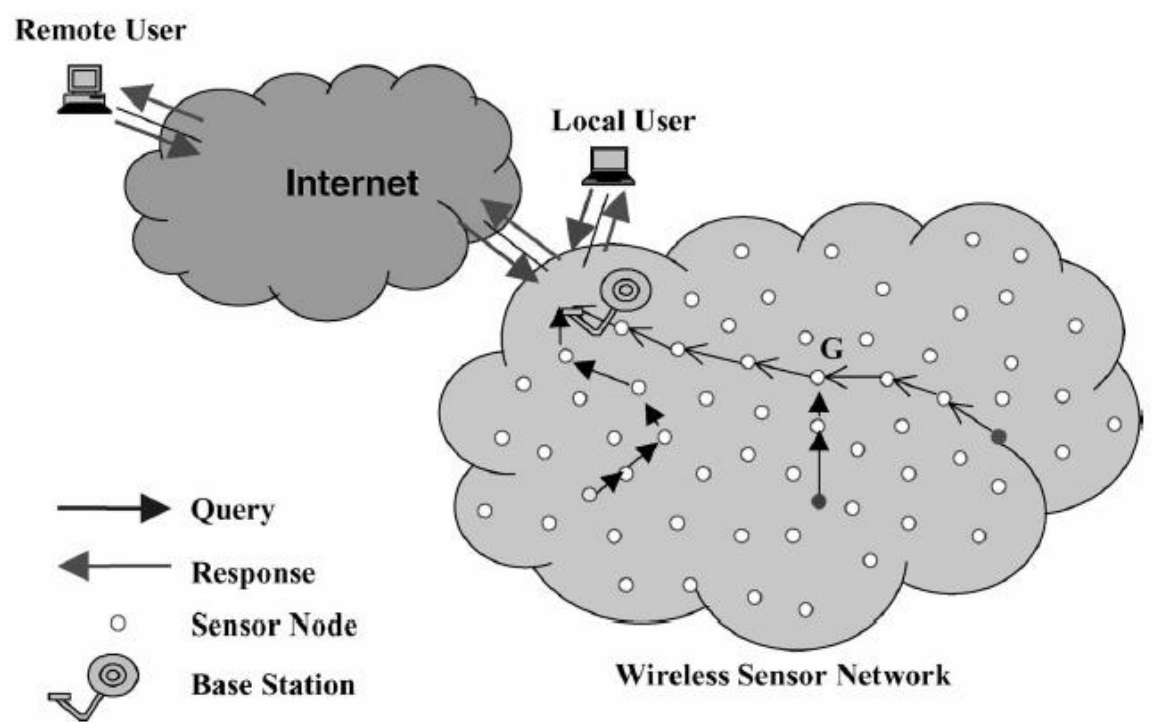

Figure 1: The way of data propagation in wireless sensor network

Although wireless sensor networks have a lot common with case and wired networks, they have unique properties which distinguish them from existing networks. These exclusive traits lead to the demands for designing a new routing different from case and wired networks. 


\section{Routing Challenges in Wireless Sensor Networks}

One of the main goals of designing wireless sensor networks is making data transmission practical so as to increase network longevity and prevent connections damages through power management methods. Routing protocols in such networks are influenced by numerous challenging factors including the followings.

1. Nodes way of arrangement

2. Energy consumption without losing accuracy

3. Data reporting modelling

4. Heterogeneity of nodes and connections

5. Faults tolerance

6. Scalability

7. Network dynamicity

8. Transmission interface

9. Connections

10. Coverage

11. Data congestion

12. Quality of services

Consequently, as stated above, routing protocols which work while being aware of the energy should provide the above requirements.

\section{Routing Approaches in Wireless Sensor Networks}

Various routing methods have been presented for wireless sensor networks so far which none of them could perform as the best and most complete approach under whole of the conditions. In fact, regarding to issues mentioned about these networks, the materials among various factors in choosing a method for routing should be always prepared.

\section{Flooding Transmission Method}

Flooding sending is a prevalent technique mostly used for discovering the route and data dissemination in case wireless networks. The routing strategy in this method does not rely on complex path detection algorithms or costly network topology maintenance. In this method, each node which receives a data packet send it for all of its neighbors. After the transition, a packet follows the entire feasible ways unless the network is disconnected, otherwise, the packet would finally reach its destination. Furthermore, in case the network topology is changed, the transferred packet will follow the new ways (Figure 2).

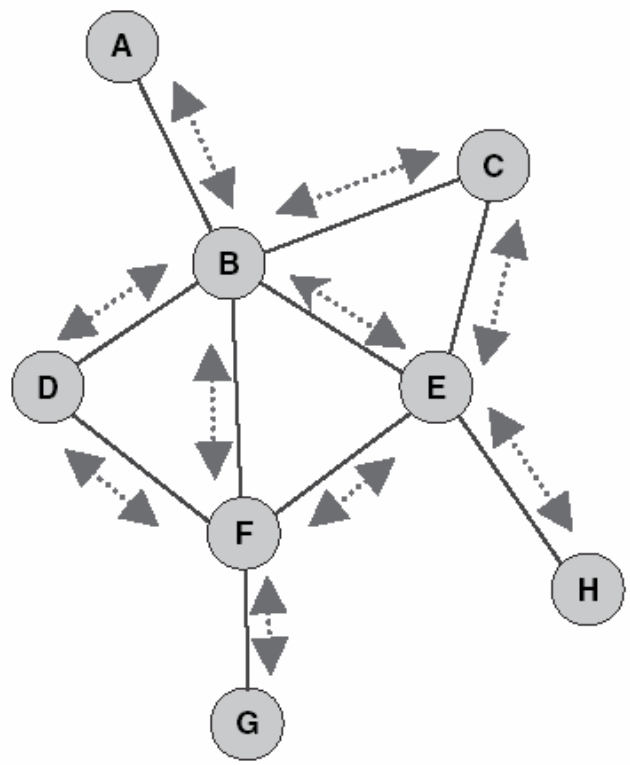

Figure 2: Principles of flooding transition method

As it can be seen in figure 2, in the simplest form, the flooding sending may lead the packets to be infinitely returned by the network nodes. In order to avoid a packet to loop in network forever, a jump number string is set in the packet normally. The first demerit of flooding transition method is its capability for traffic burst as shown in figure 3 . 


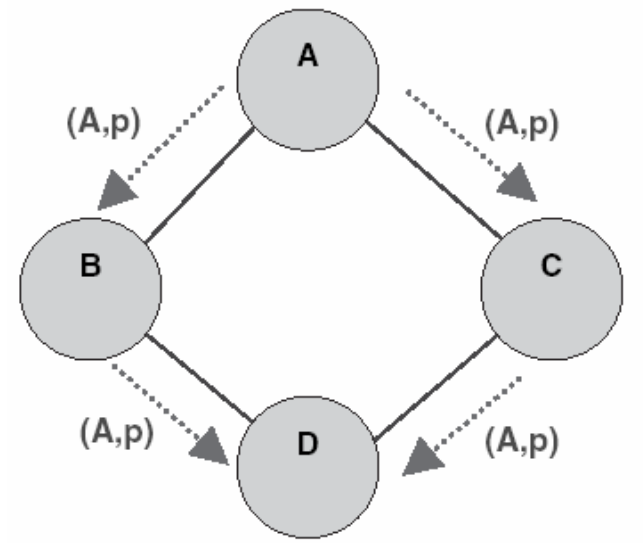

Figure 3: Traffic burst in flooding sending method

This undesirable impact is the result of sending repetitive versions of data or controlling packets to the same node. The second main disadvantage of flooding transmission is friction or overlapping. Demonstrated in figure 4 is the problem of friction and overlapping.

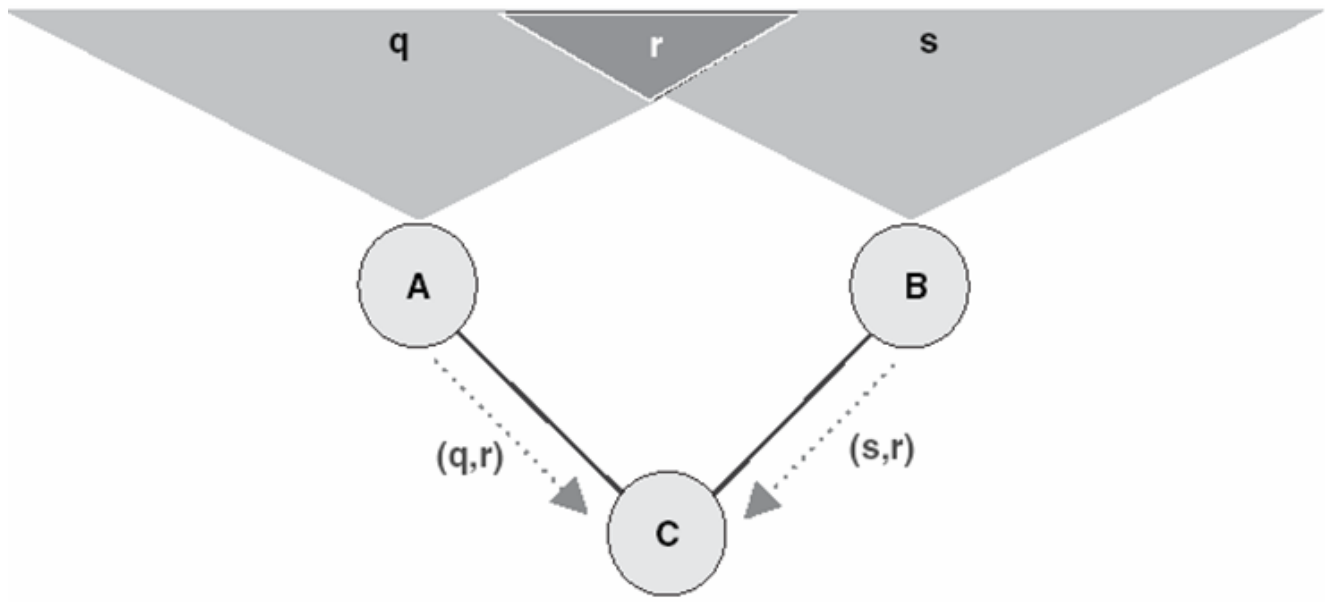

Figure 4: Friction and overlapping in flooding sending method

This problem occurs when two nodes covering the same area send packets containing the same information to the same node. The third and most important problem of flooding sending approach is sources blindness.

\section{Cluster-based Methods}

One of the most common methods proposed for data routing in wireless sensor networks is the approach based on node categorizing or clustering in other words. In this method, first, all the nodes in the network are classified into some categories according to a specific technique which in each category normally called cluster, one node is chosen as the head node and the rest of the nodes are called ordinary nodes.

The first and most known cluster-based method is LEACH ${ }^{1}$ proposed by Heinzelman et al. A threshold is used in this method in order to select the group head in each cluster. Inspired by this approach, several other methods were introduced such as X-LEACH and ImprovedLEACH.

\section{Chain-based Methods}

Another beneficial method is decreasing energy consumption based on a chain structure. In this method, at first of the network performance, all the nodes are considered as a chain structure with the minimum length and then, a node is chosen as the chain leader. This node will have the responsibility to transfer network ultimate data to the base station. PEGASIS ${ }^{2}$

\footnotetext{
${ }^{1}$ Low Energy Adaptive Clustering Hierarchy (LEACH) ${ }^{2}$ Powe Efficient Gathering in Sensor Information Systems (PEGASIS) 
chain-based method is better than LEACH approach in terms of energy consumption. However, one of the most important demerits of this technique is its high delay in data transition toward the base station.

\section{Energy Aware Methods}

Another category of tactics introduced for improving wireless sensor networks performance uses a logical assumption for its protocol. In majority of these methods named energy aware approaches, it is supposed that each sensor node is able to estimate its power source remaining energy in each moment and these methods include X-LEACH, ERA, Improved LEACH, and HEED techniques.

\section{The Role of Neural Networks in Decreasing Sensor Networks Energy Consumption}

Artificial neural network is a large system of parallel or distributed processing components called neuron or nerve cell connected to each other in a graph topology. Neurons are connected to each other using weighted connections called synapse. Artificial neural networks are math algorithms capable of learning complex mappings between input(s) and output(s) through supervised learning in addition to ranking input data using an unsupervised method. Some of these algorithms developed for neural networks can be easily adapted to the properties and demands of wireless sensor networks due to the following attributes. These features are:

Simple parallel and distributed processing

Data distributed storage

Data robustness and fault tolerance

Sensor data automatic rating

Dimensionality reduction

Low computation cost

It should be mentioned that neural networks are not energy consumption reduction methods themselves but they can be used as a smart tool by energy utilization decrease methods so as to lead these methods reach their goals more efficiently, desirably, and simply. Neural-network-based energy aware methods can be classified according to their role in neural networks, a specific network topology, or their application in energy consumption reduction plans (Table 1). Self-organizing map (SOM) neural networks have more applications in energy saving methods compared with the other neural network topologies.

Table 1: Classifying energy utilization reduction approaches based on neural networks in wireless sensor networks

\section{Neural Networks in Duty Cycle Projects}

Neural networks have been used for power dynamic management and scheduling duty cycle of sensor nodes. In this method, the time of the next event is a non-constant series approximated accurately as much as possible using Wavlet Neural networks. The mentioned neural network is actually a 3-layer grid which uses Morlet Wavelet transform in the hidden layer.

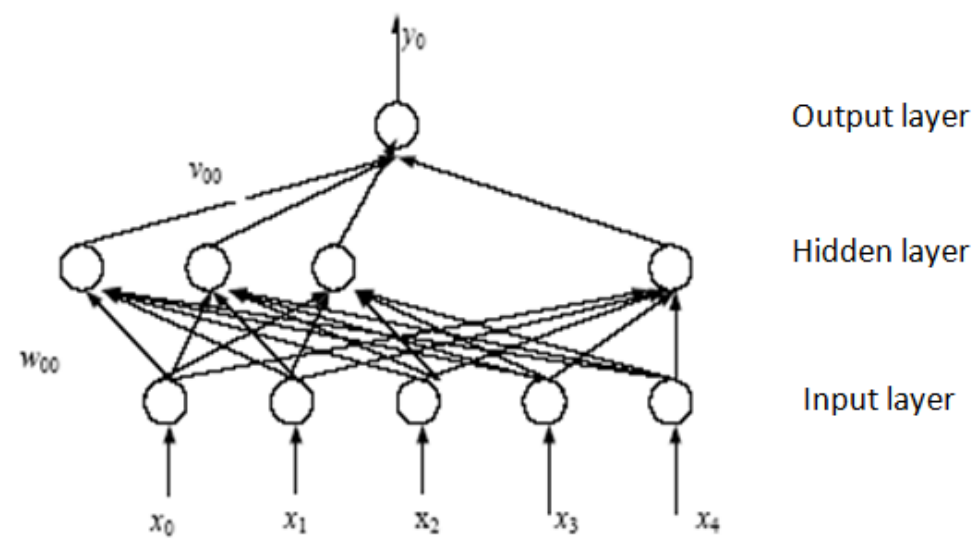

Figure 5: Wavlet neural network topologic structure Sensor data estimation through neural networks

New sensor methods have shown a great capability in reducing connections in the network through exploiting energy by forecasting sensor measurement. In these techniques, sink node extracts the time series model in order to prognosticate local readings instead of establishing direct connections with sensors and receiving real measurements. Researchers used recurrent Elman neural networks for performing the mentioned action. Moreover, second degree Newton-based methods were utilized for training this network. 


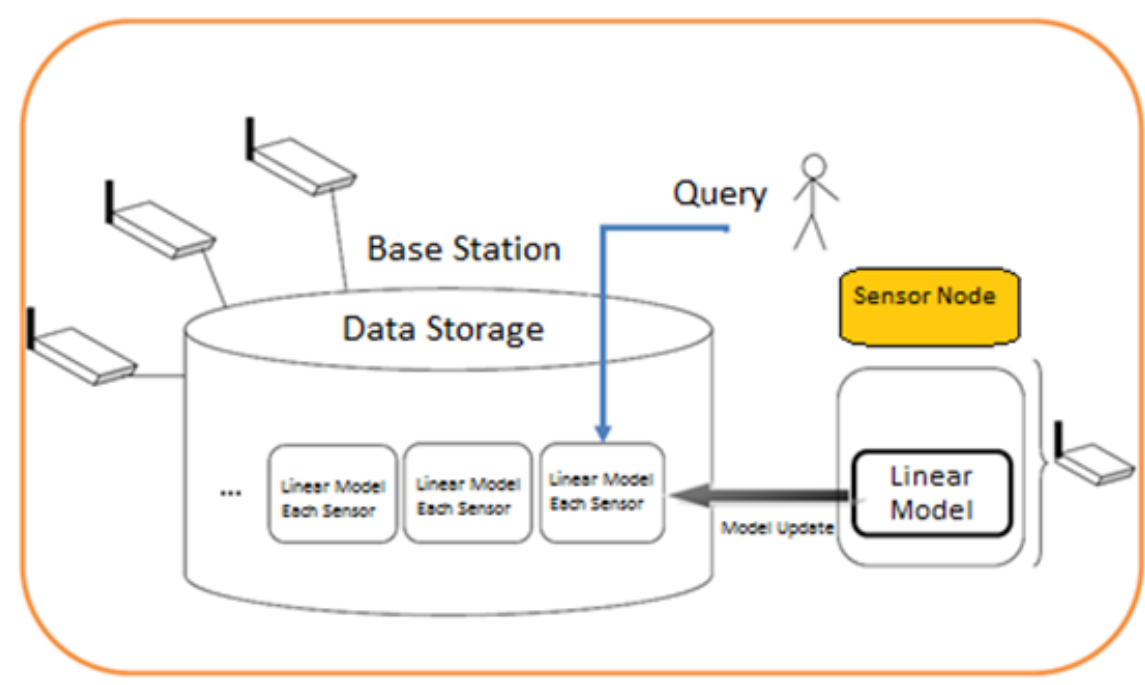

Figure 6: Sensors measurement estimation linear model

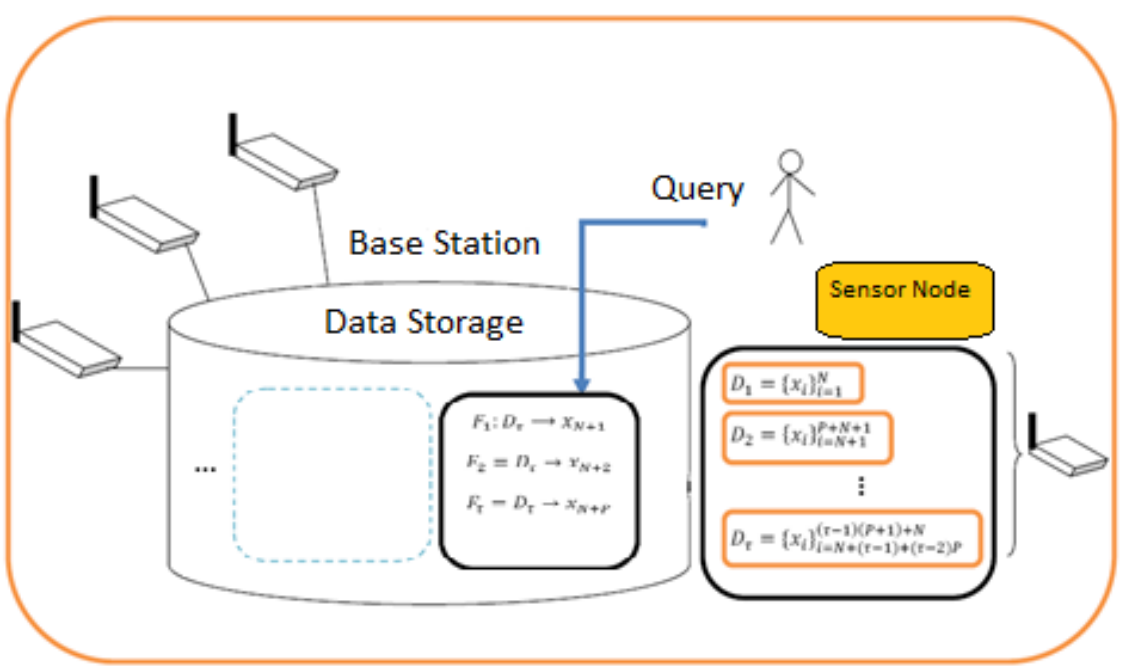

Figure 7: Sensors measurement estimation non-linear model

\section{Inter-Network Processing Using Neural Networks}

Due to the fact that computations in wireless sensor network demands less energy in comparison to connections, therefore, a considerable energy saving would be achieved through data aggregation. Data aggregation is the combination of data obtained from various sources using some functions including union, minimum selection, maximum selection, and averaging. Data aggregation is also possible through signal processing methods. In this case, it is called data fusion in which a node is able to create a more accurate signal through noise reduction and using some techniques such as wave beam forming for signals combination.

Data Association: Tracking statistics are taught to neural networks according to training methods of artificial neural networks with real or simulated values. Neural networks neutralize the effects of environmental or intentional interferences on data based on the training they have received.

Each sensor node of a Hopfield neural network has been used for storing average dependencies among the pattern components. Hopfield neural network associative memory can reproduce the damaged or lost pattern. The modified or completed pattern can be utilized for making data packets (Figure 8).

\section{Sensor Data Fusion}

One of the first usages of neural networks has been in multi-sensor data fusion for identification which has utilized a back propagation neural network (BP). 


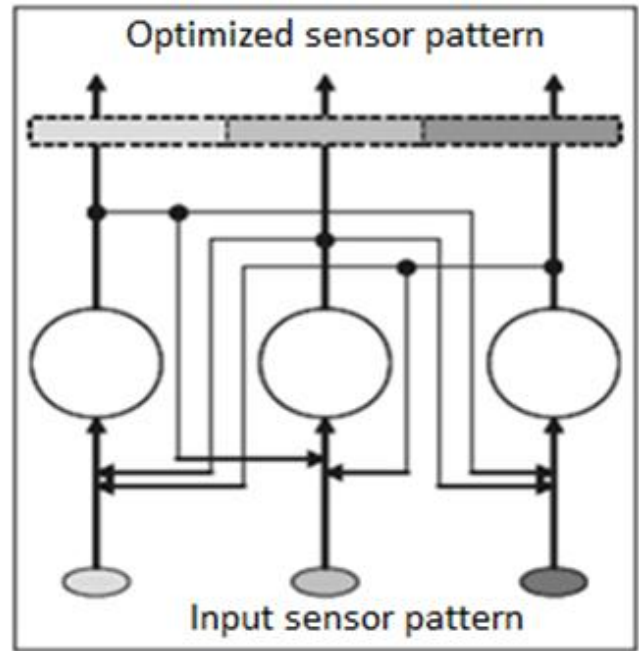

Figure 8: The structure of Hopfield neural network used in patterns modification

Back propagation networks (multi-layer networks) apparently need a long-time training and some recommendations have been proposed for the network measurement optimization. Radial basis function networks (RBF) are trained considerably faster than back propagation grids because only one layer of weights should be modified. However, this problem would no longer exist if the networks are made of the fusion between LRF and DNC.

Locating the target through collaborative sensing is furnished through fusion of several sensors data. Previous locating results are used for adaptive prediction of the target moving path. By the combination of auto regressive moving average (ARMA) model with RBF networks, the target place prognostication is done efficiently. Furthermore, productivity of a wireless sensor network would be increased through presenting an efficient organization method.

\section{Data Classification with the Environment}

Sensor nodes in an area normally create a sensing cluster and collaborate with each other by a parallel or distributed approach. SOM has been used in hierarchy network structure (cluster-based) in which the nodes have been organized in several clusters concomitant with cluster heads or data fusion centers. This selforganizing neural network reduces the data which should be transferred while ranking the similar patterns. Similar patterns classification will be done through determining the relations among patterns.

\section{Conclusion}

As mentioned, nowadays, neural networks are considered as an applicable tool in all of the energy consumption reduction fields including the duty cycle, data-oriented methods, and mobility-based approaches. With their unique capability in forecasting, ranking, identifying, and sensor data fusion as well as removing the existing challenges, neural networks can have a significant effect on wireless sensor networks energy utilization decrease in addition to increasing their operational lifetime.

\section{References}

[1] Akkaya, K. and Younis M.(2005). A Survey on Routing Protocols for Wireless Sensor Networks. Elsevier Adhoc Network Journal.325-349

[2] Alkaraki,J. N. Kamal.and A. E.(2004).On the Correlated Data Gathering Problem in Wireless Sensor Networks, Proceedings of the 9th IEEE Symposium on Computers and Communications, Alexandria, Egypt.

[3] Sohraby, K. Minoli, D.andZnati, T.(2007).Wireless Sensor Networks: Technology Protocols and Applications, Published by WILEY INTERSCIENCE.

[4] Hedetniemi, S. and Liestman, A.(1988)A Survey of Gossiping and Broadcasting in Communication Networks, IEEE Networks, Vol. 18, No. 4, 319-349.

[5] Schurgers, C. and Srivastava, M. B.(2001). Energy Efficient Routing in Wireless Sensor Networks,Proceedings of the IEEE Military Communications Conference (MilCom'01) Communications for Network-Centric Operations-Creating the Information Force, McLean, VA.

[6] Heinzelman, W. Chandrakasan, A. and Balakrishnan, H.(2000). Energy-Efficient Communication protocol for Wireless Microsensor Networks, Proceeding of the 33rd Hawaii International Conference on System Sciences (HICSS), Maui, HI.

[7] Heinzelman, W. Kulik, J.andBalakrishnan, H.(1999).Adaptive Protocols for Information Dissemination in Wireless Sensor Networks, Proceedings of the 5th ACM/IEEE International Conference on Mobile Computing and Networking (MobiCom), Seattle, WA.174-185.

[8] Heinzelman ,W. Chandrakasan, A. and Balakrishnan, H.(2002). An Application-Specific protocol Architecture for Wireless Microsensor Networks, IEEE Transactions on Wireless Communications, Vol. 1, No. 4.

[9] Handy,M.Hasse,M.andTimmermann,D.(2002).Low Energy Adaptive Clustering Hierarchy with Deterministic ClusterHead

DOI: 10.9790/0661-18040395101 $\quad$ www.iosrjournals.org $100 \mid$ Page


Selection, IEEE MWCN, Stockholm, Sweden.

[10] Xiangning,F.andYulin,S.(2007). Improvemnet on LEACH protocol of Wireless Sensor Network, In Proceedings of IEEE International Conference on Sensor Technologies and Applications, 260-264.

[11] Lindsey,S.andRaghavendra,C.(2002).PEGASIS: Power-Efficient Gathering in Sensor Information Systems, IEEE Aerospace Conference Proceedings, Vol. 3, No. 16, 1125-1130.

[12] Kofman Ravi Mazumdar,D.ShrofVivek,N.Mhatre,P.and Rosenberg, Catherine, A minimum cost heterogeneous sensor network with a lifetime. 